CERN-PH-TH/2013-006

\title{
Sensitivity of the LHC isolated $-\gamma+$ jet data to the parton distribution functions of the proton
}

\author{
L. Carminati ${ }^{1,2}$, G. Costa ${ }^{1}$, D. d'Enterria ${ }^{3}$, I. Koletsou ${ }^{1}$, \\ G. Marchiori ${ }^{4}$, J. Rojo $^{5}$, M. Stockton ${ }^{6}$, F. Tartarelli ${ }^{1}$ \\ ${ }^{1}$ INFN Sezione di Milano, Milano, Italy \\ ${ }^{2}$ Dpt Fisica, Università di Milano, Milano, Italy \\ ${ }^{3}$ CERN, PH Department, CH-1211 Geneva 23, Switzerland \\ ${ }^{4}$ LPNHE, Univ. Pierre et Marie Curie - Univ. Paris-Diderot-CNRS/IN2P3, Paris, France \\ ${ }^{5}$ CERN, PH Department, TH Unit, CH-1211 Geneva 23, Switzerland \\ ${ }^{6}$ Dept of Physics, McGill University, Montreal, Quebec - Canada
}

\begin{abstract}
We study the impact of differential isolated-photon+jet cross sections measured in proton-proton collisions at a center-of-mass energy of $\sqrt{s}=7 \mathrm{TeV}$ on the parton distribution functions (PDF) of the proton. Next-to-leading-order perturbative QCD (pQCD) calculations complemented with the NNPDF2.1 parton densities, and a Bayesian PDF reweighting method are employed. We find that although the current data provide only mild constraints to the parton densities, future $\gamma$-jet measurements with reduced experimental uncertainties can improve our knowledge of the gluon density over a wide range of parton fractional momenta $x$ as well as of the quarks at low- $x$.
\end{abstract}

\section{Introduction}

The accurate determination of the parton distribution functions (PDF) of the proton in a wide range of momentum fractions $x$ and energy scales $Q[1$ is a crucial ingredient for precision studies of the Standard Model and new physics at the Large Hadron Collider (LHC) 2,4]. The availability of new precision data from the LHC covering a large $\left(x, Q^{2}\right)$ range - including processes such as gauge boson production in association with jets and heavy quarks which hitherto have not been used for PDF determinations - provides significant improvements in the accuracy of global PDF fits [5]. Among the processes available in proton-proton $(p-p)$ collisions at the LHC, inclusive prompt- $\gamma$ production - defined as the production of photons not issuing from the electromagnetic decays of hadrons - proceeds through the dominant quark-gluon "Compton" process $q g \rightarrow \gamma q$ and has been shown to provide direct quantitative constraints on the gluon density $g\left(x, Q^{2}\right)[6]$. In this paper we revisit the phenomenological study carried out in [6] for inclusive isolated- $\gamma$ spectra [7-11], but 
focusing now on the newly available $\gamma$-jet data from the LHC [12] whose constrained kinematics given, at leading order, by the concurrent measurement of the photon and back-to-back parton, has the potential to provide additional constraints on the proton PDF. This is, to our knowledge, the first time that isolated $-\gamma+$ jet data in high-energy hadronic collisions have been used to assess their sensitivity to the proton parton densities.

\section{Experimental data}

The experimental $\gamma$-jet cross sections studied are those measured by the ATLAS experiment in $p-p$ at $7 \mathrm{TeV}$ in a data-sample corresponding to $\sim 37 \mathrm{pb}^{-1}$ integrated luminosity [12. Isolated photons have been reconstructed in the rapidity range $\left|y^{\gamma}\right|<1.37$ with a transverse energy $E_{\mathrm{T}}^{\gamma}>25 \mathrm{GeV}$, requiring a total transverse energy below $4 \mathrm{GeV}$ inside a cone of radius $\Delta R=0.4$ in pseudorapidityazimuth along the photon direction. Jets have been reconstructed with the anti- $k_{T}$ algorithm [13] with radius parameter $R=0.4$, within $\left|y^{\text {jet }}\right|<4.4$ and for transverse momenta $p_{\mathrm{T}}^{\text {jet }}>20 \mathrm{GeV}$. The differential cross sections $d \sigma / d E_{\mathrm{T}}^{\gamma}$ are then measured as a function of the photon transverse energy in six jet-photon angular configurations: $\left|y^{\text {jet }}\right|<1.2,1.2 \leq\left|y^{\text {jet }}\right|<2.8$ and $2.8 \leq\left|y^{\text {jet }}\right|<4.4$, for the same $\left(y^{\gamma} y^{\text {jet }} \geq 0\right)$ and opposite $\left(y^{\gamma} y^{\text {jet }}<0\right)$ hemispheres.

\section{Theoretical setup}

The theoretical $\gamma$-jet cross sections have been computed at next-to-leading-order (NLO) accuracy with the JETPHOX (version 1.3.0) Monte Carlo (MC) code [14 16] complemented with 100 replicas of the NNPDF2.1 parton densities 1 [17, 18. The default renormalisation, factorisation and fragmentation scales are all set equal to the photon transverse energy, $\mu_{\mathrm{R}}=\mu_{\mathrm{F}}=\mu_{\mathrm{ff}}=E_{\mathrm{T}}^{\gamma}$. The parton-to-photon fragmentation functions used are the BFG-II ("large gluon") set [19. The MC photon isolation and jet reconstruction criteria are matched as closely as possible to each of the experimental cuts. The $\gamma$-jet distributions have been corrected with PYTHIA [20] for non-perturbative effects due to hadronization and $p-p$ underlying event.

In order to quantify the impact of the photon-jet cross sections on the PDF, we use the Bayesian reweighting method described in Refs. [21,22] (the same technique could have been performed using PDF sets based on Hessian error matrices, such as CT10 23] and/or MSTW08 24], as discussed in [25]). We compare each of the experimental distributions (with $N_{\text {dat }}$ data points) to the theoretical prediction obtained with each of the $k=1, \ldots, N_{\text {rep }}=100$ replicas of the NNPDF2.1 set, defining a data-theory goodness-of-fit $\chi_{k}^{2}$ as

$$
\chi_{k}^{2}=\frac{1}{N_{\mathrm{dat}}} \sum_{i=1}^{N_{\mathrm{dat}}} \frac{\left(\sigma_{i}^{(\mathrm{th}),(k)}-\sigma_{i}^{(\mathrm{exp})}\right)^{2}}{\Delta_{\text {tot }}^{2}},
$$

where $\sigma_{i}^{(\mathrm{th}),(k)}$ is the NLO theoretical prediction for the photon-jet cross section obtained with the $f_{k}$ PDF replica, $\sigma_{i}^{(\exp )}$ is the corresponding experimental measurement, and $\Delta_{\text {tot }}$ accounts for the

\footnotetext{
${ }^{1}$ Use of the more recent NNPDF2.3 set [5], which includes LHC data but which came available only after this analysis was performed, is expected to give the same conclusions as our study.
} 
experimental statistical and systematic uncertainties added in quadrature2. Theoretical uncertainties due to scale variations are between $15 \%$ (low $E_{\mathrm{T}}^{\gamma}$ ) and $10 \%$ (high $E_{\mathrm{T}}^{\gamma}$ ) - as obtained from the envelope of the theoretical spectra obtained varying the 3 scales in the following 6 combinations: $\left(\mu_{\mathrm{R}}, \mu_{\mathrm{F}}, \mu_{\mathrm{ff}}\right) / E_{\mathrm{T}}^{\gamma}=(1 / 2,1,1)(2,1,1)(1,1 / 2,1)(1,2,1)(1,1,1 / 2)(1,1,2)(1 / 2,1 / 2,1 / 2)(2,2,2)-$ but have not been included in the $\chi^{2}$ analysis as there is not yet a recipe to consistently include scale uncertainties in global PDF analysis. The corresponding weight of each replica is then obtained following [21,22]. These weights $w_{k}$, divided by the number of MC replicas of the prior PDF set $\left(N_{\text {rep }}\right)$, give the probabilities of the replicas $f_{k}$ given the $\chi_{k}^{2}$ values to the newly added experimental results.

For each set of weights we also compute the rescaling parameter $\alpha$ [21, 22, which indicates the value by which one should scale the experimental and/or theoretical uncertainties in order to achieve a goodness-of-fit $\chi_{k}^{2} \approx 1$. The distribution of the rescaling variable $\alpha$, normalized to unity, is used to investigate if any potential disagreement between NLO pQCD and a given dataset could be due to possibly over-/under-estimated uncertainties, and/or indicate possible tensions with other datasets.

The dependence of the measured $\gamma$-jet cross sections on the individual flavour of the underlying parton densities can be quantified by computing the correlation coefficient between each of the light-quark and gluon distributions and the NLO cross sections [17]. These correlations are shown in Figs. 1 and 2 for various configurations of the $\gamma$-jet production at the LHC and for two values of the photon transverse energy: $E_{\mathrm{T}}^{\gamma}=27.5 \mathrm{GeV}$ and $E_{\mathrm{T}}^{\gamma}=300 \mathrm{GeV}$ respectively. Photons+jets at central rapidities (top panels) have a dominant sensitivity to $g\left(x, Q^{2}\right)$ around $x=0.01$ for low $E_{\mathrm{T}}^{\gamma}$, and around $x=0.1$ for high $E_{\mathrm{T}}^{\gamma}$. At forward jet rapidities (bottom panels) isolated- $\gamma$ probe the gluon and light-quark densities for a wide range of values at medium and small- $x$ for small and moderate $E_{\mathrm{T}}^{\gamma}$, while for the highest $E_{\mathrm{T}}^{\gamma}$ the light quarks are probed at very large- $x$.

\section{Results}

\subsection{Analysis of current LHC photon-jet data}

Figure 3 shows the ratios between the experimental and theoretical $d \sigma / d E_{\mathrm{T}}^{\gamma}$ differential cross sections for the six jet rapidity ranges of the ATLAS measurement. The (yellow) band gives the range of the predictions obtained for each of the 100 replicas, while the outer error-bars cover the sum in quadrature of the statistical and (asymmetric) systematic uncertainties of the measurement. We observe an overall good agreement for all rapidity ranges within uncertainties, except for the most forward jets $\left(2.8 \leq\left|y^{\text {jet }}\right|<4.4\right)$ where the data have a more concave shape than the theory, and in the lowest bin $\left(E_{\mathrm{T}}^{\gamma}=27 \mathrm{GeV}\right)$ where the theoretical predictions overshoots the central value of the data points (a trend already observed in a few of the LHC inclusive isolated- $\gamma$ spectra [6]). Figure 4 shows the comparison of the measured photon-jet distributions with the NLO predictions obtained using the central NNPDF2.1 replicas, as a function of $x=E_{\mathrm{T}}^{\gamma}\left(e^{\left\langle y^{\mathrm{jet}}\right\rangle}-e^{-\left\langle y^{\gamma}\right\rangle}\right) / \sqrt{s}$. The six data/theory ratios are around unity indicating an overall good agreement between NLO pQCD and the experimental measurements over the range $x \approx 10^{-2}-1$.

\footnotetext{
${ }^{2}$ The full experimental covariance matrix is not available for this measurement - the usefulness of future $\gamma$-jet data would be increased if this covariance matrix is provided (see discussion in Section 4.2).
} 

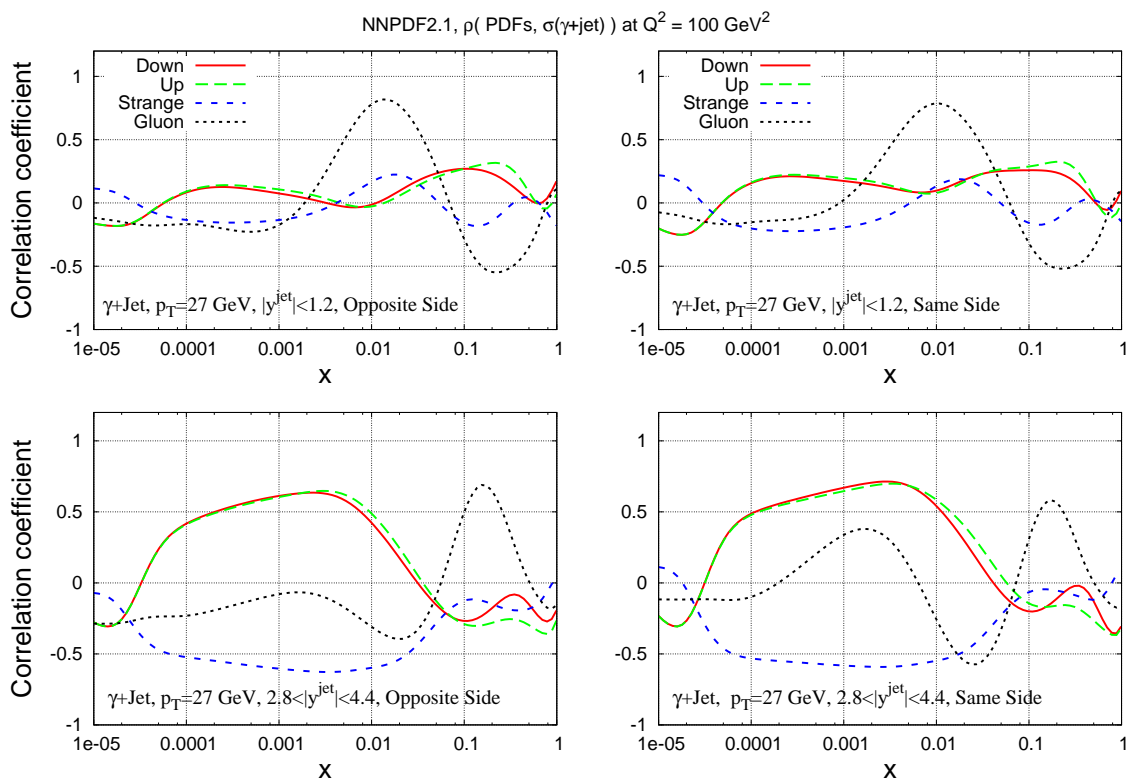

Figure 1: Correlations between the $\gamma$-jet cross section in $p$-p collisions at $7 \mathrm{TeV}$ (for the smallest value of the photon transverse energy, $E_{\mathrm{T}}^{\gamma}=27.5 \mathrm{GeV}$ ) and various flavours of the NNPDF2.1 parton densities for central $y^{\text {jet }}$ (top) and forward $y^{\text {jet }}$ (bottom) for the opposite (left) and same-side (right) photon-jet hemispheres.
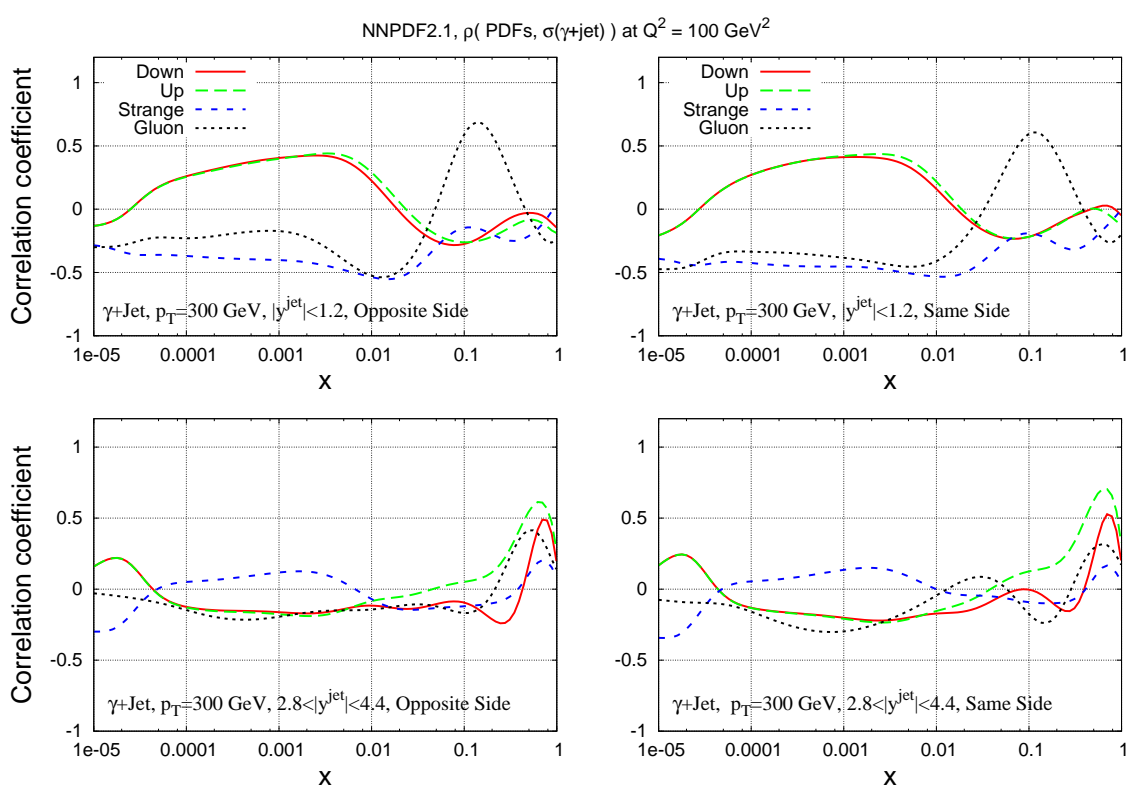

Figure 2: Correlations between the $\gamma$-jet cross section in $p$ - $p$ collisions at $7 \mathrm{TeV}$ (for the largest values of the photon transverse energy, $E_{\mathrm{T}}^{\gamma}=300 \mathrm{GeV}$ ) and various flavours of the NNPDF2.1 parton densities for central $y^{\text {jet }}$ (top) and forward $y^{\text {jet }}$ (bottom) for the opposite (left) and same-side (right) photon-jet hemispheres. 

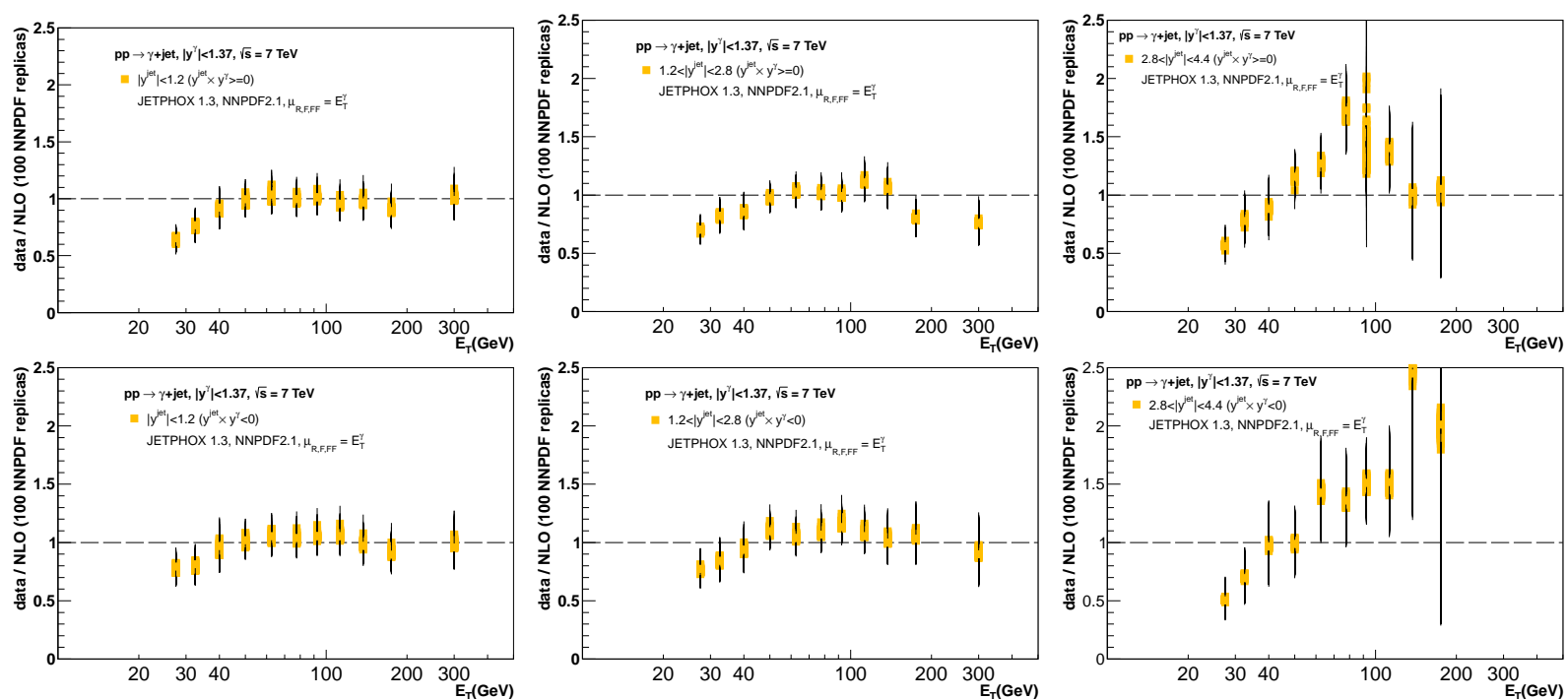

Figure 3: Ratio of the ATLAS photon-jet $E_{\mathrm{T}}^{\gamma}$-differential cross sections over NLO pQCD predictions in $p$ - $p$ collisions at $\sqrt{s}=7 \mathrm{TeV}$ for the quoted jet rapidities. The top (bottom) panels show the case where photons and jets are produced in the same (opposite) hemispheres. The (yellow) band indicates the range of predictions for each of the 100 NNPDF2.1 replicas, and the bars show the total experimental uncertainty.

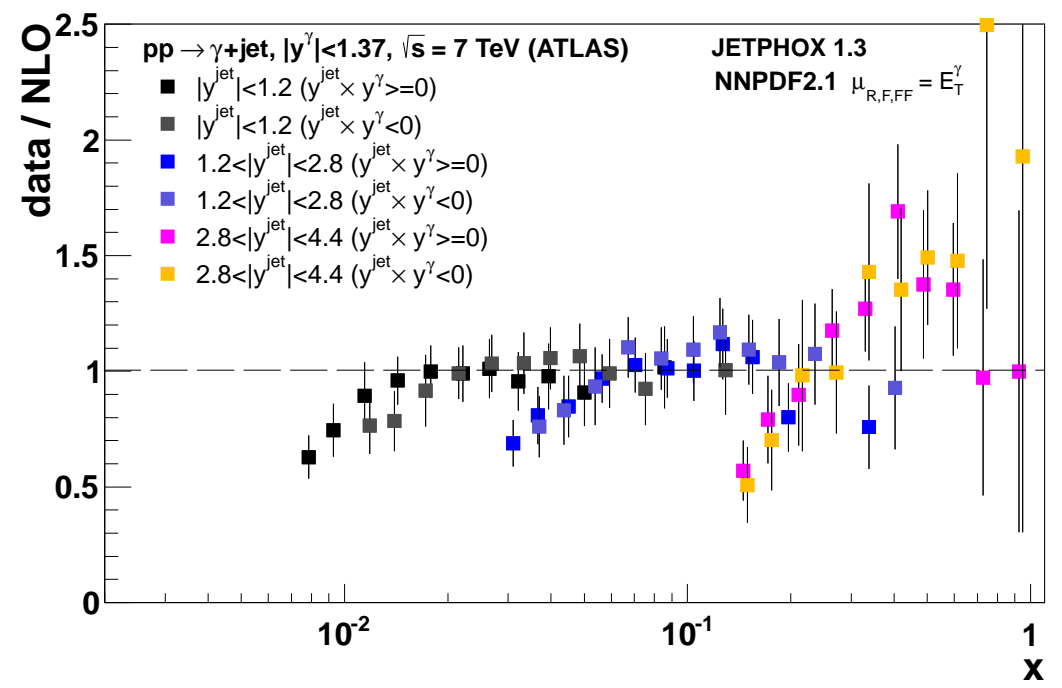

Figure 4: Data/theory ratios for the six photon-jet distributions considered in this analysis, as a function of $x=E_{\mathrm{T}}^{\gamma}\left(e^{\left\langle y^{\mathrm{jet}}\right\rangle}-e^{-\left\langle y^{\gamma}\right\rangle}\right) / \sqrt{s}$. For each system, the NLO prediction used is the one obtained with the central NNPDF2.1 replica. The error bars indicate the total experimental uncertainty. 
Table 1 summarizes the quantitative results of our study. The first column lists the average $\chi^{2}$ between each of the datasets and the NLO calculations over all replicas. For a majority of cases the agreement is quite good $\left(\chi^{2} \approx 1\right)$, while for the most forward jet configurations the $\chi^{2}$ obtained is poorer $\left(\chi^{2} \approx 1.9\right.$ and $\chi^{2} \approx 2.7$ in the $\gamma$-jet opposite and same-hemisphere cases respectively). This result confirms at the quantitative level that there is an overall good agreement between NLO pQCD and the experimental isolated $-\gamma+$ jet spectra measured at the LHC, as found previously for all the inclusive isolated $-\gamma$ distributions [6].

Table 1: Summary of the $\chi^{2}$-analysis between NLO pQCD and the ATLAS $\gamma$-jet data. For each system we list the initial data-theory $\chi^{2}$ (and its associated standard deviation), the $\chi_{\mathrm{rw}}^{2}$ obtained after including each corresponding dataset via PDF reweighting, the mean $\langle\alpha\rangle$ of the associated $\mathcal{P}(\alpha)$ distribution, and the effective final number of replicas after reweighting.

\begin{tabular}{cc|c|c|c|c}
\hline Rapidities & $\gamma$-jet $y$-hemisphere & $\chi^{2} \pm \sigma_{\chi^{2}}$ & $\chi_{\mathrm{rw}}^{2}$ & $\langle\alpha\rangle$ & $N_{\text {eff }}$ \\
\hline$\left|y^{\text {jet }}\right|<1.2,\left|y^{\gamma}\right|<1.37$ & same & $1.1 \pm 0.2$ & 1.2 & 1.2 & 98 \\
$\left|y^{\text {jet }}\right|<1.2,\left|y^{\gamma}\right|<1.37$ & opposite & $0.7 \pm 0.3$ & 0.5 & 0.8 & 96 \\
$\left|y^{\text {jet }}\right|=1.2-2.8,\left|y^{\gamma}\right|<1.37$ & same & $1.3 \pm 0.1$ & 1.2 & 1.2 & 98 \\
$\left|y^{\text {jet }}\right|=1.2-2.8,\left|y^{\gamma}\right|<1.37$ & opposite & $0.7 \pm 0.2$ & 0.6 & 0.9 & 99 \\
$\left|y^{\text {jet }}\right|=2.8-4.4,\left|y^{\gamma}\right|<1.37$ & same & $2.7 \pm 0.6$ & 2.6 & 1.9 & 81 \\
$\left|y^{\text {jet }}\right|=2.8-4.4,\left|y^{\gamma}\right|<1.37$ & opposite & $1.9 \pm 0.3$ & 1.9 & 1.5 & 96 \\
\hline
\end{tabular}

In Fig. 5 we show the $\mathcal{P}(\alpha)$ distributions for the various kinematical configurations. In all cases $\mathcal{P}(\alpha)$ peaks close to one apart from the most forward jet data-samples, confirming the overall consistency of these datasets with NLO pQCD and the proper estimation of the associated experimental errors. Since experimental uncertainties seem correctly determined for the measurement, the $\langle\alpha\rangle \approx 1.5-2$ value of the most-forward jets results may point to larger theoretical uncertainties in this region due to some inadequacy of fixed-order NLO calculations for such a kinematical configuration. Similar differences between data and theory for forward-central dijet distributions in $p-p$ collisions have been observed at $7 \mathrm{TeV}[26$. Excluding the same-side large-rapidity data, the total initial $\chi^{2}$ of all the systems considered is $\chi^{2}=1.1$, while after reweighting it decreases to $\chi_{\mathrm{rw}}^{2}=1.0$. The corresponding total effective number of replicas after reweighting is $N_{\text {eff }}=97$.

The direct quantification of the impact on the gluon and light-quark distributions is shown in Fig. 6] where the ratios of NNPDF2.1 NLO PDF, evaluated at $Q^{2}=100 \mathrm{GeV}^{2}$, are plotted before and after including the ATLAS isolated $-\gamma+$ jet data. The central values of the NLO parton densities are essentially unaffected by the new photon-jet LHC data which only lead to a rather mild (about $5 \%) \mathrm{PDF}$ uncertainty reduction at intermediate gluon fractional momenta $x \approx 0.06$ to 0.3 and in the small- $x$ region between $10^{-4}$ and $10^{-2}$ for light quarks. 

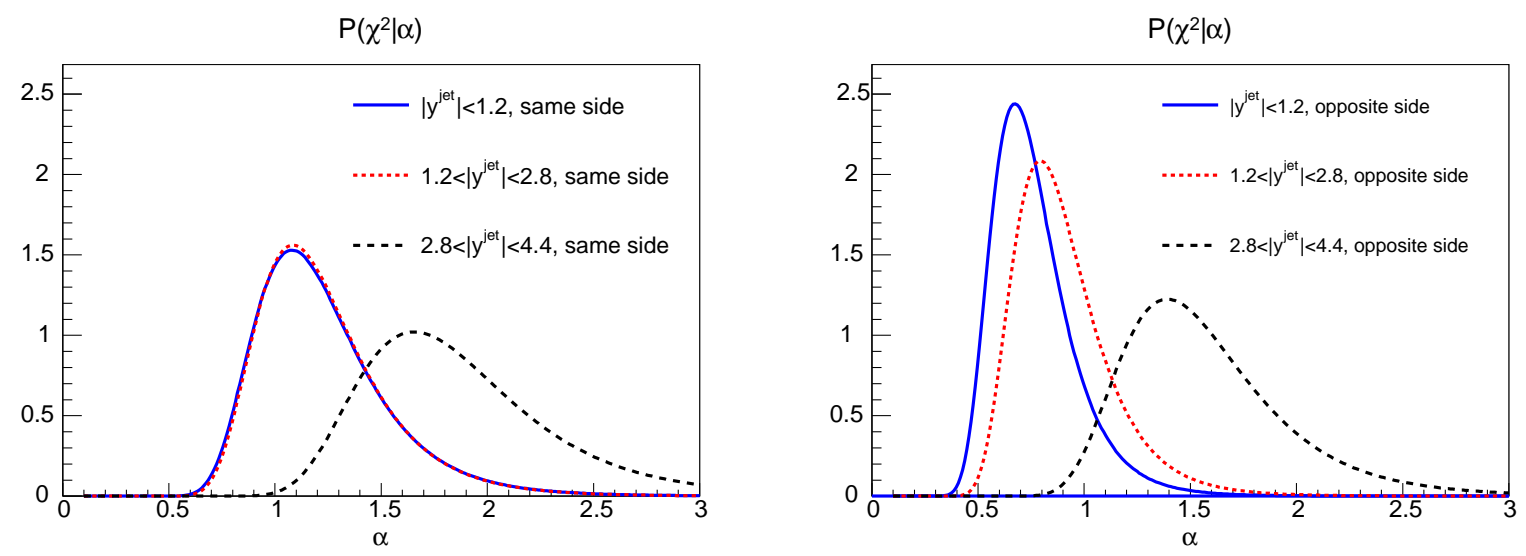

Figure 5: Distribution of the $\alpha$ rescaling variable for the six jet rapidity bins of the ATLAS $\gamma$-jet data in $p-p$ collisions at $\sqrt{s}=7 \mathrm{TeV}$. The left (right) plot shows the results for the same (opposite) $\gamma$-jet $y$-hemisphere.

\subsection{Analysis of LHC photon-jet pseudodata with reduced uncertainties}

The current relatively large experimental uncertainties of the available LHC photon-jet measurements, as well as the lack of availability of their associated covariance error matrix, result in a limited impact on the improvement of our knowledge of the proton PDF. To quantify the possible sensitivity of future more precise LHC $\gamma$-jet data, we have generated pseudodata for the same kinematics of the ATLAS measurement, based on the NNPDF2.1 central predictions and assuming a total uncorrelated experimental uncertainty of $\pm 5 \%( \pm 10 \%)$ for central and forward jets and of $\pm 8 \%$ ( $\pm 15 \%$ ) for very-forward jets, above (below) $E_{\mathrm{T}}^{\gamma}=45 \mathrm{GeV}$ respectively. Such a scenario represents a realistic improvement of about a factor of 2 with respect to the current measurement. We generate the pseudodata adding Gaussian random fluctuations and carry out the same PDF reweighting analysis done with real data. The resulting ratios of reweighted over current NLO PDF are shown in Fig. 7 for the simulated, more precise, $\gamma$-jet pseudodata. We observe an improved sensitivity to the gluon and quark PDF in particular, for the latter, at small- $x$. The PDF uncertainties are reduced by up to $20 \%$ in some $x$ regions. This result indicates that the inclusion of future differential photon-jet cross sections $d \sigma / d E_{\mathrm{T}}^{\gamma}$ data into global PDF analyses has the potential to reduce the uncertainties of the gluon and light-quarks densities.

\section{Summary}

We have quantified the impact on the proton PDF of the existing isolated- $\gamma+$ jet $E_{\mathrm{T}}^{\gamma}$-differential cross sections in $p-p$ collisions at $7 \mathrm{TeV}$ where the photons are measured at central rapidities $\left(\left|y^{\gamma}\right|<1.37\right)$ and the jets over $\left|y^{\text {jet }}\right|<4$.4. Our theoretical setup includes NLO pQCD theoretical calculations as implemented in the JETPHOX program combined with the NNPDF2.1 parton densities and its associated PDF reweighting technique. We find that NLO pQCD provides a good description of the photon-jet results at the LHC in a wide kinematic range of photon transverse energies and jet rapidities, except maybe for the events where the jets are emitted at the most forward rapidities. The systematic uncertainties of the available measurements are however still too large 

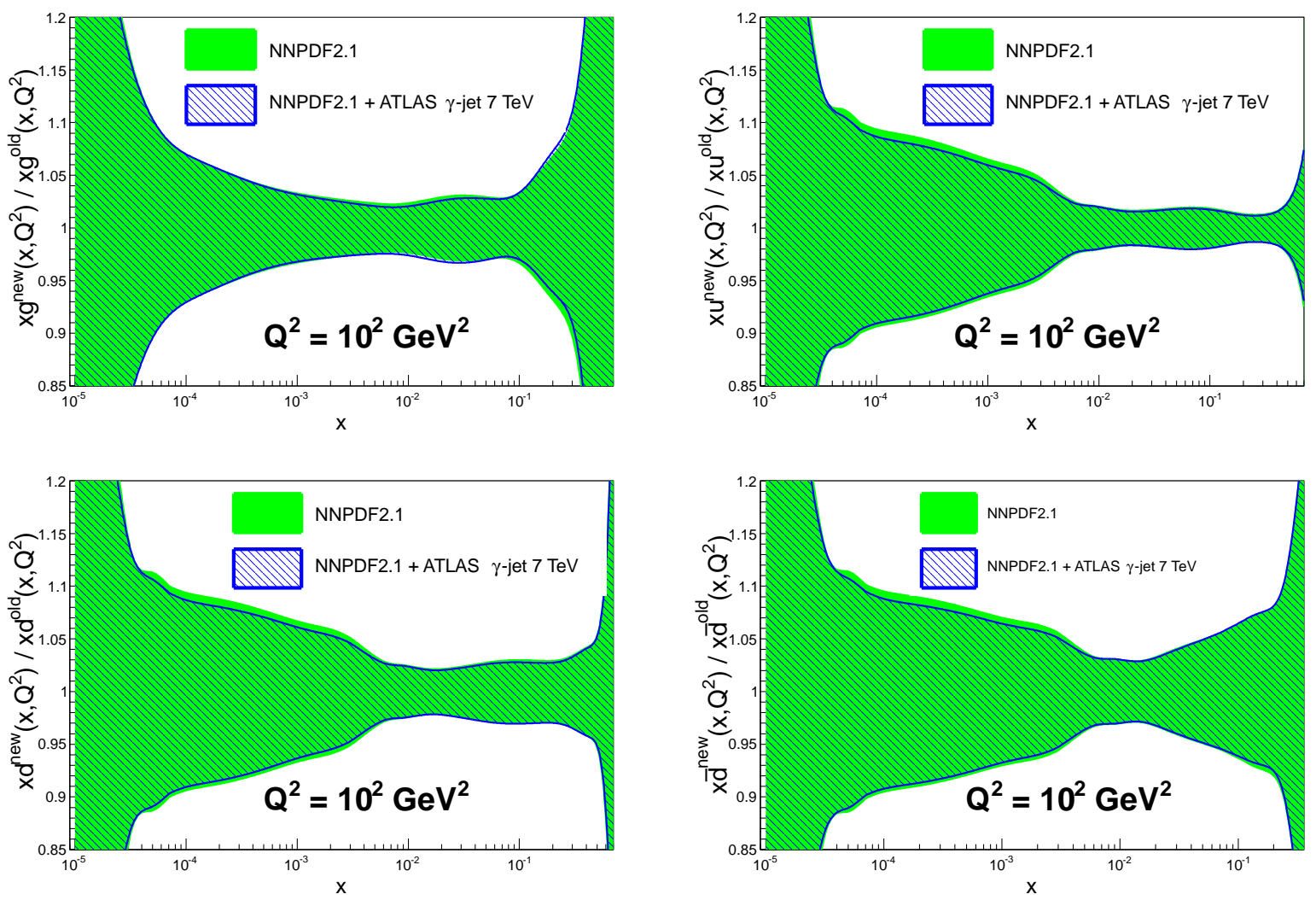

Figure 6: Ratio between the NNPDF2.1 NLO PDF and associated uncertainties before (green solid band) and after (dashed blue area) inclusion of the ATLAS $\gamma$-jet data measured at 7 TeV. From top to bottom and from left to right we show the gluon, the up quark, the down quark and the anti-down quark. PDF are valued at $Q^{2}=100 \mathrm{GeV}^{2}$.

to provide significant constraints on the proton PDF. Nonetheless, our quantitative studies with pseudodata confirm that future $\gamma$-jet measurements with reduced uncertainties can indeed provide constraints on both the gluon density over a large $x$ domain as well as on the small- $x$ light-quarks distributions. Photon-jet measurements at the LHC, as already shown in previous similar studies for inclusive isolated prompt- $\gamma$, constitute thus an interesting ingredient of future global PDF fits, complementary to the other data-sets currently used.

\section{Acknowledgments}

The research of J. R. has been supported by a Marie Curie Intra-European Fellowship of the European Community's 7th Framework Programme under contract number PIEF-GA-2010-272515. 

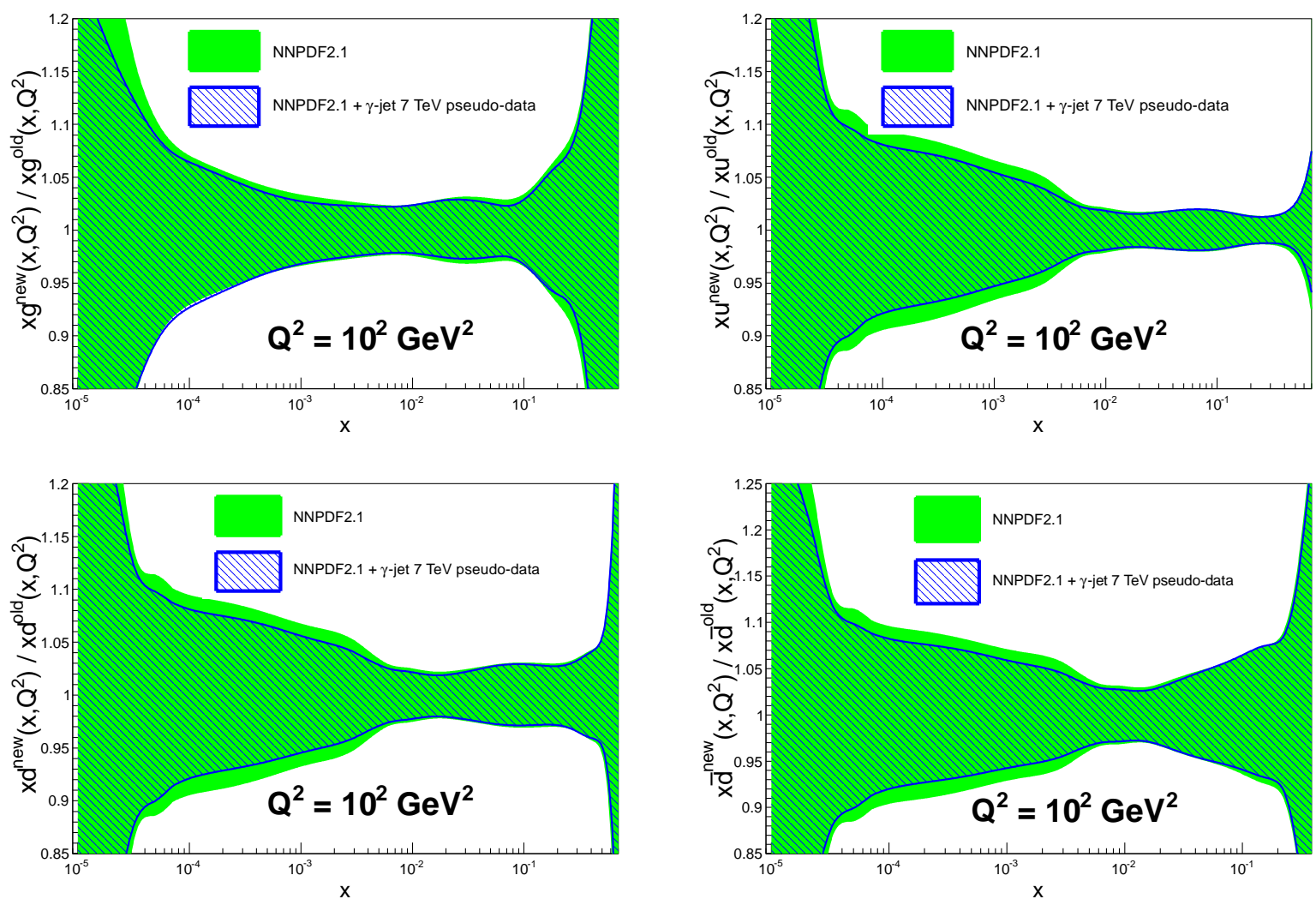

Figure 7: Same as Fig. 6 in the case of artificial $\gamma$-jet pseudodata at $7 \mathrm{TeV}$ assuming the same kinematical distributions of the existing ATLAS measurement but with reduced experimental uncertainties. 


\section{References}

[1] E. Perez and E. Rizvi, Rep. Prog. Phys. 76 (2013) 046201, arXiv:1208.1178.

[2] G. Watt, JHEP 1109 (2011) 069, arXiv:1106.5788.

[3] NNPDF Collab., R.D. Ball et al., (2012), arXiv:1211.5142.

[4] S. Forte, Acta Phys.Polon. B41 (2010) 2859, arXiv:1011.5247.

[5] NNPDF Collab., R.D. Ball et al., Nucl.Phys. B867 (2013) 244, arXiv:1207.1303.

[6] D. d'Enterria and J. Rojo, Nucl.Phys. B860 (2012) 311, arXiv:1202.1762.

[7] CMS Collab., V. Khachatryan et al., Phys. Rev. Lett. 106 (2011) 082001, arXiv:1012.0799.

[8] ATLAS Collab., G. Aad et al., Phys. Rev. D83 (2011) 052005, arXiv:1012.4389.

[9] CMS Collab., S. Chatrchyan et al., Phys. Rev. D84 (2011) 052011, arXiv:1108.2044.

[10] CMS Collab., S. Chatrchyan et al., Phys.Lett. B710 (2012) 256, arXiv:1201.3093.

[11] ATLAS Collab., G. Aad et al., Phys. Lett. B706 (2011) 150, arXiv:1108.0253.

[12] ATLAS Collab., G. Aad et al., Phys.Rev. D85 (2012) 092014, arXiv:1203.3161.

[13] M. Cacciari, G.P. Salam and G. Soyez, JHEP 0804 (2008) 063, arXiv:0802.1189.

[14] P. Aurenche et al., http://lappweb.in2p3.fr/lapth/PHOX_FAMILY/jetphox.html.

[15] P. Aurenche et al., Nucl. Phys. B399 (1993) 34.

[16] S. Catani et al., JHEP 05 (2002) 028, hep-ph/0204023.

[17] NNPDF Collab., R.D. Ball et al., Nucl. Phys. B849 (2011) 296, arXiv:1101.1300.

[18] NNPDF Collab., R.D. Ball et al., Nucl. Phys. B855 (2012) 153, arXiv:1107.2652.

[19] L. Bourhis, M. Fontannaz and J.P. Guillet, Eur. Phys. J. C2 (1998) 529, hep-ph/9704447.

[20] T. Sjostrand, S. Mrenna and P.Z. Skands, JHEP 05 (2006) 026, hep-ph/0603175.

[21] NNPDF Collab., R.D. Ball et al., Nucl. Phys. B849 (2011) 112, arXiv:1012.0836.

[22] NNPDF Collab., R.D. Ball et al., Nucl. Phys. B855 (2012) 608, arXiv:1108.1758.

[23] H.L. Lai et al., Phys. Rev. D82 (2010) 074024, arXiv:1007.2241.

[24] A.D. Martin et al., Eur. Phys. J. C63 (2009) 189, arXiv:0901.0002.

[25] G. Watt and R. Thorne, JHEP 1208 (2012) 052, arXiv:1205.4024.

[26] CMS Collab., S. Chatrchyan et al., JHEP 1206 (2012) 036, arXiv:1202.0704. 